\title{
PREFACE: VIRUS-RELATED AND MEDICAL SPRAYS
}

We are delighted to present this special issue of Atomization and Sprays dedicated to "Virusrelated and Medical Sprays." Recently, deeper understanding of the physical mechanisms of atomization processes has led to a number of major technological developments, some examples of which are described in articles in this special issue.

Since the outbreak of the COVID-19, there has been enormous public interest in subjects that our research community considers "mainstream," combined with various topics/aspects specifically related to the current pandemic. Such combinations are reflected in the studies presented here- among them, the physics behind the disintegration and spreading of droplets that are exhaled and the degree of dispersal of these droplets and ligaments that may contain various viruses and pathogens. This kind of knowledge provides the basis for understanding the risk associated with virus transfer in various enclosures. Indeed, the inhalation of droplets is no less important than exhalation, and, hence, attention is focused on both aspects, viz. the inhalation of the virus, as well as drugs inhaled during treatment of patients.

As guest editors, we would like to thank all authors who contributed to this special issue and the Editors-in-Chief, who enabled us to assemble it.

Guest Editors:

David Katoshevski

Environmental Engineering Unit

Safety Management and Engineering Unit

Ben-Gurion University of the Negev

Israel
Tali Bar-Kohany

School of Mechanical Engineering

The Iby and Aladar Fleischman Faculty of Engineering

Tel-Aviv University

Israel 
